\title{
The Application of Digital Prepress Technology in Advertising Project
}

\author{
Xingguo Xu \\ Guangxi Technological College of Machinery and Electricity, Nanning Guangxi, 530007, China
}

Keywords: Digital prepress technology, Workflow, Advertising project, Application.

\begin{abstract}
This article aims to explore the application of digital prepress technology in the production of advertising projects. The research method is a brief introduction and an example of the basic principles in prepress process. It discusses the design, input, output and printing process involved in the design process of advertisement project. It provides pre-press technical process and Technical reference.
\end{abstract}

\section{The basic workflow of digital prepress technology}

\section{Digital prepress manuscript processing method}

High-quality print on the picture accuracy requirements

High-quality advertising prints on the accuracy of the original requirements of clear and delicate picture is pleasing to the eye.

1. Image resolution setting

Advertising print image resolution of not less than 300dpi appropriate. When scanning pictures, set the output resolution to the resolution of the image transferred from the scanner to Photoshop. In Photoshop, you can also use the "image size" command to modify the resolution. Download the picture from the Internet, a resolution of 72dpi, can not be directly used for digital preprint layout, the implementation of "image> image size" command, call out the "image size" dialog box, cancel the "re-image pixels" check, will distinguish The rate is changed to 300 pixels / inch, and then check the "reset image pixels" to confirm the "constraint ratio" of the check is activated, the "print size" to the corresponding value.

2. Select color modes

In the graphic image design software, the color mode of the image is RGB mode, CMYK mode, gray mode and other color modes. RGB images are bright but can not be used directly for output, because the color gamut is larger than the CMYK color mode, the color can be displayed on the screen, but the four-color ink does not print out. Image For digital media display, the color mode of the image is best to choose RGB mode because the RGB mode is more vivid, richer and clearer. If the image is black and white, you should select the grayscale mode. Before printing a board, the picture must use the CMYK mode or the grayscale mode. In the Photoshop settings, the CMYK mode or the grayscale mode is selected via the "Image> Mode" menu.

3. Adjust image sharpness

Appropriate use of the sharpening function in the image improves the clarity of certain patches of picture boundaries. Sharpness appropriate, the image appears clear; sharpness is low, it is blurred. Photoshop provides sharpening tools and sharpening filters to a certain extent to improve the local quality of the image, but not a large area to improve the quality of the image, nor for the image to add real details.

Image source and image processing

1. Printed matter 
When the customer provides a printed original, the designer should confirm whether there is a photo of the original, if not should try to reduce the dot. Using the "variegated" speckle filter in Photoshop can reduce the dot, but obviously blur the image, as the scanner's "go to the net" effect is good.

\section{Photograph}

There is no network of photos, no need to go to the network scan. When scanning high-quality photos, pay attention to maintaining the image layering. In the dark image of the image is very boring, or bright part of the glare, layering is not as good as the original, it is necessary to adjust the scan settings.

\section{Paintings}

In response to the high quality of the painting set, the requirements are completely faithful to the color of the original drawing, but the scanned image will be slightly off color, need to pass the calibration screen, adjust the color drawing original in Photoshop. If the scanner can not be swept over, it is necessary to block the scan, and then the puzzle, so that the image of convergence natural, complete.

\section{Pattern and logo}

The pattern and logo samples provided by the customer will show a uniform or regular gradation, with a clear outline, so the designer can re-paint in the graphics software to make it clear than the original to accommodate the various layouts.

\section{Color reversal film}

Color reversal film has a good sense of hierarchy, clarity, after amplification still maintain high quality, in the production of fine print, it is a class of important originals. The scanner that configures the transmission function can scan the reversal film, but to ensure the quality of the image, it is better to use an electrical extension to handle the reversal film.

\section{Digital Imaging}

The image size of a digital camera is measured in terms of "pixel size". From the pixel size value, you can calculate its size at $300 \mathrm{~d}$ resolution.

7. Use the Internet to download images

For high-end print design using the Internet to download the image, you need to download the relevant professional material website, such as: material China, red moving China, thousands of network and other sites, we must pay attention to the image pixel size and compression quality.

Types of text manuscripts and their handling

1. Typing, copying, faxing, printing originals

The use of text recognition software can quickly deal with these materials, require clear writing, and background contrast significantly. The text printed on paper is actually some graphics. The text recognition software converts it into plain text stored in the computer.

\section{Pure text electronic document}

Pure text electronic document that is TXT format files, can be PC "Notepad" or Apple's "SimpleText" can also be guided to open a variety of typesetting software, safe and convenient to use. So a variety of electronic text, should be converted to TXT format, and then spread to the designer's computer. In order to avoid the various documents into simple text in the process of typographical errors, loss of images, but also the original print out for inspection, proofreading.

\section{Other formatted files}

HTML document page format may also contain pictures, layout settings, text attribute settings and other factors, before use, copy the text to "Notepad", or in Internet Explorer to implement "File> Save As" in the "Save as" Select "Text File", proofread, print original as control. PDF document with AcrobatReader open, and then copy the text on the page to "Notepad", converted into plain text.

A variety of typesetting files Some customers will have done past prepress documents, asked for revision, this manuscript is made with professional typesetting software, if you use a different software typesetting. You should first copy the text in the original document to the PC "Notepad". Convert to plain text, and then import your typography software. 


\section{Advertising Project Design}

The process of advertising project design is a planned, step-by-step process of continuous improvement, the success of the design depends largely on the concept is accurate, consider whether it is perfect.

\section{Investigation}

Investigation is to understand the process of things, the design is to have a purpose, is the cultural background, market research, industry surveys and other information on the brand, audience, product information, positioning, performance practices, survey is the design basis.

\section{Contents}

Content is divided into subject content and specific content of two parts, which is the designer in the design of the first-hand materials.

\section{Idea}

Conception is the first step in the design. The idea has always been independent of the design. In the designer's visual work to convey the idea of a product or business, so as to better product positioning.

\section{Visual elements}

In the design, the constituent elements become a combination of designer works, each element has the function of transmitting information. In the use of color, can reflect a designer of the color of understanding and cultivation. Color is a language, color with feelings, people can produce associations, people can produce cold, before and after, the severity, the size of the sense.

\section{Performance method}

In the three major composition, there are many kinds of graphics processing and expression techniques, such as contrast, analogy, exaggeration, symmetry, primary and secondary, light and shade, variation, repetition, contradiction, radiation, rhythm, thickness, well-being, area and other forms.

\section{Balance}

Balance can bring visual and psychological satisfaction. Designers to solve the picture of the "force field" balance, before and after the convergence of the balance, the sense of balance is also the designer needs, the balance and imbalance is relative to the subject requirements as the standard. Balance is divided into symmetrical balance and asymmetric balance, including point, line, surface, color, space balance. Performance method to create a visual excitement to sublimation works.

\section{Advertising project production}

\section{Check and processing}

After the design is finalized, the external link of the image is released in CorelDraw, all the text is converted to the path, the image is checked and processed in the aspects of image, page setting, bleeding, font, color mode, etc., and the layout file is saved as a CorelDraw file. Board file. Routine inspection and processing of images is done in Photoshop, mainly for color adjustment and image patching, color adjustment preparation - prepress coloring, routine checking and handling of content in typesetting files.

\section{Digital proofing}

\section{Digital proofing technology}

The so-called digital proofing, is the color desktop system to produce the page data, without any form of simulation means, directly through the color printer output proofs to check the prepress process 
image page quality, for the printing process to provide proofs, and provide customers with You can sign the basis of printing. Now digital proofing system can not only output FM network structure and continuous tone of the proofs, but also can be output and follow-up the actual printing exactly the same amplitude modulation structure of proofs. So that even if the color separation sheet has not yet been output or the printing plate has not been printed, you can see from the proof of the actual printing effect. Compared with the traditional proofing technology, digital proofing technology has the following characteristics and advantages:

1.Color image reproduction performance

The color image reproduction performance includes the tone range of the image, the density or chroma of the field or saturated color, the gray balance, the reduction of the gradation curve, including the bright tone, the middle tone, the dark tone level reproduction and the network expansion rate reproduction and the delicate texture Wait. Color management software is to measure the need to simulate the printing of the standard file color and the printer with the standard file color block, respectively, ICC format data, the color management system software calculation, the establishment of proofing process required characteristics of the calibration file Profile. In this way, PS, TIFF format file RIP after the data, as long as sent to the digital proofing system, you can output and follow-up printing match the proofs.

Traditional proofing technology, because the proofer in speed, pressure, embossing and other aspects are different from the actual printing, so the traditional proofing is difficult to simulate the actual printing, printing is also difficult to catch up with the effect of traditional proofing, and digital proofing proofs in reality In use, the printer operator is generally more likely to simulate, because digital proofing and printing in the entire color space in the color difference is less than the traditional proofing and printing between the color.

\section{Resolution ratio}

As the digital proofing system is usually used inkjet printing or laser printing technology, the general output of the FM network or continuous tone structure, so as long as there are more than 600dpi output resolution, proofing proofs can achieve the effect of amplitude modulation dot 150lpi. Now most color printers can achieve such image resolution.

3.Stability of sample output

As the digital proofing system is sent directly from the digital page file to the proofing system, before the output proof, all by the digital signal control and transmission, so whenever the output, the same electronic file output is exactly the same. Of course, this stability is the premise of color printer hardware performance, such as inkjet ink droplets size, ink and printing I can wait to be consistent.

For the traditional offset printing technology, in addition to paper, ink, PS version should remain stable and the state of mechanical proofing equipment, proofing process connected to the proof of the field density can not be consistent, let alone also depends on the level of the operator as a factor. Compared with the traditional proofing technology, digital proofing is almost free from the environment, equipment, technology and other aspects of the impact, not affected by the operator, its stability, consistency is very satisfactory.

4.Output speed

Digital proofing system output speed has been the technology can popularize the bottleneck. The large format, high-resolution inkjet printer on the market, the output of a large open $(102 \mathrm{~cm} \times 78 \mathrm{~cm})$ 720dpi proof of the time, it takes 40 minutes or more, which does not include RIP interpretation of the time. The reason why digital pickup speed is significantly improved mainly depends on the development of multi-nozzle inkjet proofing technology and fast RIP proofing and server, and some proofing server can control several digital proofing machine at the same time.

\section{Proofing format}

General high-performance digital proofing system for the A3 format. With the inkjet printer hardware resolution and speed of the gradual increase in the capacity of the cartridges increased, constantly changing the application of ink cartridges, large format output of the inkjet printer 
endlessly, now has a width of 1.5 meters above the digital proofing System, a variety of format models can simulate a variety of printing machine amplitude effect.1.6 System cost

Traditional proofing system not only requires expensive proofing equipment, but also supporting the proofing room, air conditioning equipment, etc., but also need to output color separation, copy of the branch, proofing costs are considerable. The digital proofing system hardware only color printers, control computers and supporting RIP and color management software. With the reduction of the cost of ink, the promotion of special printing paper, will also use ordinary paper after surface treatment in the inkjet printer output, then the cost of digital proofing system may be reduced. As a result of no output dichroic film, copy, mechanical proofing and other processes, not only greatly shorten the prepress design, production, proofing the total cycle, saving a lot of raw materials.

6. Requirements for technical staff

Traditional mechanical proofing requires experienced, high-quality operators, in the amount of work, the need to shift shift substitutions, which will not only bring labor costs increase, but also become a proof of sample quality of the unstable factors. The digital proofing system as long as the production of designers know how to use the correct proofing computer can be, digital proofing system can be 24 hours a day to start running.

\section{Conclusions}

This paper expatiates on the application of digital prepress technology in advertising project, and expatiates on the data processing and exchange of digital prepress technology from design, scanning input, document processing, digital proofing, output, printing and even postpress A great effect. The future of digital prepress technology will be completed in the process of the system, the development of toner and inkjet technology will be partially replaced by offset, gravure and flexographic printing, the future will affect the magazine, newspaper industry, consumables and printing service system.

\section{References}

[1] Hu Weiyou, Digital Printing and Computer Direct Plate Making Technology, Printing Industry Press, 2011.08.

[2] Su Chunrang, Digital Image Map Pre - press Plate Making Technology and Application, Printing quality and standardization. 2011 Vol.3.

[3] Shen Zhiwei, On use of CTP system, Digital Printing, 2008, Vol.8.

[4] Li Wei, The Main Imaging Technology and Application in Modern Print Media Production, Printing Technology, 2003 Vol.31.

[5] Jin Yang, Present Situation and Development of Flexible Plate Plate Making Technology, Digital Printing, 2004 Vol.2.

[6] Li Zhijiang, Liu Quanxiang. Lithography technology. Beijing:Printing Industry Press, 2007. 